\title{
Prevalencia de enfermedades neuro-músculo esqueléticas en la Comunidad de San Eduardo
}

\section{Prevalence of neuro-musculoskeletal diseases, in the Community of San Eduardo Prevalência de doenças neuro-músculoesqueléticas na Comunidade de San Eduardo}

\author{
Dayanna Lisbeth Rodríguez Bravo \\ dayanna.rodríguez@cu.ucsg.edu.ec \\ https://orcid.org/0000-0003-4055-801X
}

\author{
Isabel Odila Grijalva Grijalva \\ isabel.grijalva@cu.ucsg.edu.ec \\ https://orcid.org/0000-0003-4491-4149
}

\author{
Jhoana Muñóz Linares \\ jhoana.muñoz@cu.ucsg.edu.ec \\ https://orcid.org/0000-0003-4743-2540 \\ Jorge Enrique Soria Ruíz \\ jorge.soria@cu.ucsg.edu.ec \\ https://orcid.org/0000-0001-5445-8111
}

\author{
Mónica del Rocio Galarza Zambrano \\ monica.galarza@cu.ucsg.edu.ec \\ https://orcid.org/0000-0001-9694-7773 \\ Universidad Católica de Santiago de Guayaquil, Guayaquil-Ecuador \\ Recibido 18 de marzo 2021 | Arbitrado y aceptado 12 de abril 2021 | Publicado en 4 de mayo 2021
}

\section{RESUMEN}

Las enfermedades músculo esqueléticas son alteraciones del aparato locomotor. Término que engloba un conjunto de afectaciones de origen congénito, traumático o patológico, que desencadenan deficiencias, dolencias y trastornos motores. Objetivo. Determinar la prevalencia de enfermedades neuro-músculo esqueléticas en la Comunidad de San Eduardo. Metodología. El trabajo de investigación tuvo un alcance descriptivo, enfoque cuantitativo de corte transversal y carácter deductivo. Para el estudio se utilizó el instrumento RFT 5-33, que contiene cinco secciones, las cuatro primeras identificaron a la familia, por datos de ubicación; rol que desempeñó el participante; tipo y composición familiar; riesgos individuales; antecedentes patológicos familiares y la quinta sección, el perfil de los miembros de la comunidad Resultados. La edad según el sexo predominó de 2.759 personas, un $75 \%(n=2069)$ a mayores de 42 años; un $25 \%(n=690)$ a menores de 13 años; edad promedio de 28,6 años, una mediana de 25 años y máxima de 99 años de los cuales 1.388 (50,3 \%) hombres y 1.371 (49,7 \%) mujeres, que no representaron diferenciación significativa con respecto al sexo. Con respecto a la prevalencia de enfermedades neuro-músculo esqueléticas, se determinó a la lumbalgia en un $26 \%$; artritis el $16 \%$; osteoporosis 14,1\%; artrosis en un 13,6 \% y en menor porcentaje el trastorno neurológico central en un $7 \%$ relacionada a enfermedad mental y epilepsia. Conclusión. Las enfermedades músculo esqueléticas, que prevalecieron fueron la lumbalgia, artritis, osteoporosis y artrosis, de acuerdo a la base de datos del Proyecto Familias y Comunidades Saludables.

Palabras clave: Prevalencia; enfermedades; sistema nervioso; músculo esquelético; comunidad

\begin{abstract}
Musculoskeletal diseases are alterations of the locomotor system. A term that encompasses a set of congenital, traumatic or pathological disorders that trigger deficiencies, ailments and motor disorders. objective. To determine the prevalence of neuro-musculoskeletal diseases in the Community of San Eduardo. Methodology. The research work had a descriptive scope, a cross-sectional quantitative approach and a deductive character. For the study, the RFT 5-33 instrument was used, which contains five sections, the first four identified the family by location data; role played by the participant; family type and composition; individual risks; family pathological history and the fifth section, the profile of community members. Results. Age according to sex predominated in 2,759 people, $75 \%(n=2,069)$ over 42 years old; $25 \%(n=690)$ to children under 13 years of age; average age of 28.6 years, a median of 25 years and a maximum of 99 years of which 1,388 (50.3\%) were men and 1,371 (49.7\%) were women, who did not represent significant differentiation with respect to sex. Regarding the prevalence of neuromusculoskeletal diseases, low back pain was determined in 26\%; arthritis 16\%; osteoporosis 14.1\%; osteoarthritis in $13.6 \%$ and in a lower percentage the central neurological disorder in $7 \%$ related to mental illness and epilepsy. Conclusion. The musculoskeletal diseases that prevailed were low back pain arthritis, osteoporosis and osteoarthritis, according to a database from the Healthy Families and Communities Project.
\end{abstract}

Key words: Prevalence; diseases; nervous system; skeletal muscle, community

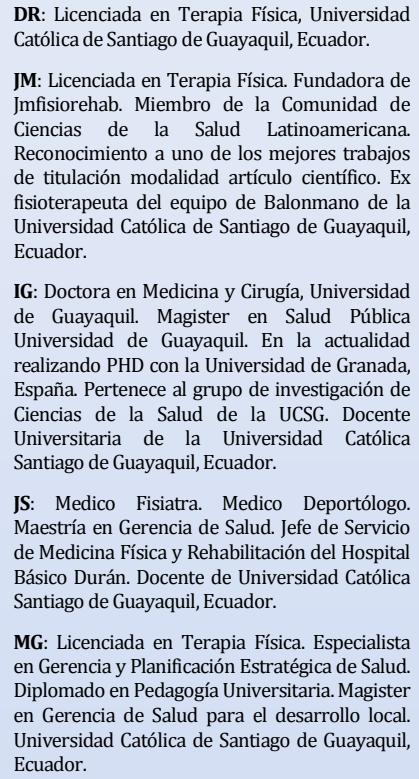
de Guayaquil. Magister en Salud Pública Universidad de Guayaquil. En la actualidad
realizando PHD con la Universidad de Granada, realizando PHD con la Universidad de Granada, España. Pertenece al grupo de investigación de Ciencias de la Salud de la UCSG. Docente
Universitaria de la Universidad Católica Santiago de Guayaquil, Ecuador.

JS: Medico Fisiatra. Medico Deportólogo. Maestría en Gerencia de Salud. Jefe de Servicio de Medicina Física y Rehabilitación del Hospital Básico Durán. Docente de Universidad Católica Santiago de Guayaquil, Ecuador.

MG: Licenciada en Terapia Física. Especialista en Gerenciay Planificación Estratégica de Salud. Diplomado en Pedagogía Universitaria. Magister en Gerencia de Salud para el desarrollo local. Universidad Católica de Santiago de Guayaquil, Ecuador. 
DR: Licenciada en Terapia Física, Universidad Católica de Santiago de Guayaquil, Ecuador.

JM: Licenciada en Terapia Física. Fundadora de Jmfisiorehab. Miembro de la Comunidad de Ciencias de la Salud Latinoamericana Reconocimiento a uno de los mejores trabajos Reconociento a uno de los mejores trabajos fisioteraperta del equo de Balona de fisioterapeuta del equipo de Balonmano de la Santiago de Guayaquil, Ecuador.

IG: Doctora en Medicina y Cirugía, Universidad de Guayaquil Magister en Salud Pública de Guayaquil. Magister en Salud Pública Universidad de Guayaquil. En la actualidad realizando PHD con la Universidad de Granada, España. Pertenece al grupo de investigación de Ciencias de la Salud de la UCSG. Docente Universitaria de la Universidad Católica Santiago de Guayaquil, Ecuador.

JS: Medico Fisiatra. Medico Deportólogo. Maestría en Gerencia de Salud. Jefe de Servicio de Medicina Física y Rehabilitación del Hospital Básico Durán. Docente de Universidad Católica Santiago de Guayaquil, Ecuador.

MG: Licenciada en Terapia Física. Especialista en Gerencia y Planificación Estratégica de Salud. Diplomado en Pedagogía Universitaria. Magister en Gerencia de Salud para el desarrollo local. Universidad Católica de Santiago de Guayaquil, Ecuador.

\begin{abstract}
RESUMO
As doenças musculoesqueléticas são alterações do aparelho locomotor. Termo que engloba um conjunto de distúrbios congênitos, traumáticos ou patológicos que desencadeiam deficiências, enfermidades e distúrbios motores. Objetivo. Determinar a prevalência de doenças neuro-musculoesqueléticas na Comunidade de São Eduardo. Metodologia. 0 trabalho de investigação teve um âmbito descritivo, uma abordagem quantitativa transversal e um carácter dedutivo. Para o estudo, foi utilizado o instrumento RFT 5-33, que contém cinco seções, as quatro primeiras identificaram a família por dados de localização; papel desempenhado pelo participante; tipo e composição da família; riscos individuais; história patológica familiar e a quinta seção, o perfil dos membros da comunidade. Resultados. A idade segundo o sexo predominou em 2.759 pessoas, $75 \%(n=2.069)$ acima de 42 anos; $25 \%(n=690)$ para crianças menores de 13 anos; idade média de 28,6 anos, mediana de 25 anos e máximo de 99 anos sendo 1.388 (50,3\%) homens e 1.371 (49,7\%) mulheres, o que não representou diferenciação significativa em relação ao sexo. Em relação à prevalência de doenças neuro-musculoesqueléticas, a lombalgia foi determinada em 26\%; artrite $16 \%$; osteoporose 14,1\%; osteoartrite em 13,6\% e em menor porcentagem o distúrbio neurológico central em 7\% relacionado a doenças mentais e epilepsia. Conclusão. As doenças musculoesqueléticas mais prevalentes foram lombalgia, artrite, osteoporose e osteoartrite, segundo banco de dados do Healthy Families and Communities Project.
\end{abstract}

Palavras-chave: Prevalência; doenças; sistema nervoso; músculo esquelético; comunidade

\section{INTRODUCCIÓN}

$\mathrm{L}$ a OMS indica que a nivel mundial existe una problemática de salud, que abarca signos y síntomas que dificultan la ejecución de "Actividades Básicas de la Vida Diaria, Instrumentales y Avanzadas". En el 2017, se recopila información sobre la realidad que conlleva este tipo de limitación en la movilidad, destreza y capacidades funcionales. Siendo el dolor lumbar más frecuente; incrementando diagnósticos que superan el $20 \%$ y $33 \%$ de casos, convirtiéndose en fuente de origen para desarrollo de múltiples afectaciones relacionadas a patologías músculo esqueléticos (1-2).

Los problemas neurológicos, son adquiridos por miles de personas en el mundo. Aproximadamente el $80 \%$ de defunciones están presentes en países de bajos o medianos recursos, afectando al sistema nervioso central y periférico, en otras palabras, involucrado el cerebro, medula espinal, raíces nerviosas y músculos. Los daños en mayor medida son accidentes cerebrovasculares, epilepsia y demencia, restringiendo funcionalidad motora, sensitiva y cognitiva que repercuten en el bienestar global, generando un problema a largo plazo (3).

La prevalencia en desórdenes músculo esqueléticos, mostró que el motivo de las alteraciones estructurales tenía relación con repetición constante de carga física, y el desplazamiento. La fuerza de manipulación existente del cuello, espalda dorsal y hombros representan el 48,1\% de zonas físicas con mayor afectación en población colombiana. Esto demuestra que 
la columna vertebral es donde se acumula la mayor parte del peso y potencia externa que repercuten hacia otros segmentos corporales (4).

Otros estudios realizados en Perú, muestran que las enfermedades músculos esqueléticas están presente en mujeres que en hombres, el mayor número de casos localizados en zona baja de la espalda en un 6,76 \%; muñeca y mano 5,57 $\%$. Además, se reportó la frecuencia de dolor que dentro de primeros siete días se asocia con síntomas de estructuras orientadas a patologías reumáticas altamente incapacitantes; que generan deficiencia, limitación de funcionabilidad y realización de actividades cotidianas (5).

Las enfermedades neurológicas representan el 10,64 \% de población peruana; siendo factores de riesgo que disminuye estándares de vida. Se encontró más predominante en mujeres, porque las afectaciones se asocian a trastornos incapacitantes, que se relacionan con la edad y otros factores adquiridos (6). Las alteraciones nerviosas y trastornos neurodegenerativos son principales diagnósticos de etapas avanzadas. En la población colombiana el 56,9 \% de participantes son mujeres, diagnosticadas con demencia no específica. El 31,4 \% demencias leves; un 9,2\% traumatismos; $5,4 \%$ discapacidad de origen neurológico que engloban perdida del funcionamiento cognitivo, relevante en limitación de actividades y desenvolvimiento de participación social (7).

Según un estudio de la Secretaría de Salud Laboral y Medio Ambiente del gobierno Español, (2019) demuestra que los trastornos músculo esqueléticos de origen laboral, son los producidos en tendones, músculos y articulaciones, huesos, cartílagos, ligamentos y nervios.
Teniéndose en cuenta que los coordina el sistema nervioso y que dicho conjunto óseo-muscular a la vez puede influir indirectamente en el sistema circulatorio, digestivo y respiratorio. Siendo la lumbalgia, tendinitis, epicondilitis, hernias, cervicalgias y síndrome del túnel carpiano. Al ser una gama de dolencias tan extensa, los trabajadores deben ser informados sobre los riesgos específicos para evitar este tipo de dolencias, según los puestos de trabajo y tareas que se tengan que realizar para concienciar y sensibilizar. Como se detallan en las estadísticas europeas los daños más frecuentes se localizan en la espalda en un $25 \%$ de la población trabajadora y extremidades superiores como cuello-hombros, codos y muñecasmanos (8).

Los investigadores Camacho, Esteban y Paradas indican que las enfermedades neuromusculares son las que afectan al sistema nervioso periférico, al músculo esquelético, a la unión neuromuscular y la médula espinal. El conjunto de trastornos es muy numeroso y se clasifican en función de la localización de la lesión: enfermedades de motoneurona, radiculopatías, plexopatías, neuropatías, enfermedades de la unión neuromuscular y por último, enfermedades musculares. Dentro de cada grupo, el origen puede ser metabólico, infeccioso, tóxico, inmunomediado, genético 0 neurodegenerativo. El comienzo acontece tanto en la infancia como en la edad adulta, y el curso evolutivo es variable: agudo, subagudo o crónico. El diagnóstico y el tratamiento de la patología neuromuscular requieren una alta especialización (9).

En el Ecuador en el año (2013), se realizó una investigación relacionada a los "Síntomas Músculoesqueléticos en Trabajadores Operativos del Área de 
Mantenimiento de una Empresa Petrolera Ecuatoriana". Dejando como resultado una mayor prevalencia de síntomas músculoesqueléticos, en el grupo de trabajadores de entre 30 y 40 años de edad, en las regiones anatómicas: espalda baja 66 $(64,7 \%)$, seguido de espalda alta 44 $(43,1 \%)$, cuello $38(37,3 \%)$ y hombro 27 $(26,5 \%)$, siendo los más afectados los puestos de trabajo técnico-eléctrico y técnico-mecánico (10).

En la Comunidad de San Eduardo, se desconoce la prevalencia de enfermedades neuro-músculoesqueléticas, debido a diversos factores asociados $y$ sociodemográficos, como causas inminentes de los problemas que afrontan los habitantes de la Cooperativa 25 de Julio y Virgen del Cisne.

El análisis de la investigación contempla la necesidad de identificar los factores asociados de este sector vulnerable debido a que la actividad económica es irregular, acompañado de factores ambientales que no son de óptimas condiciones y considerando que la comunidad no se encuentra sobre cimientos adecuados, afectando la condición y calidad de vida de las personas que habitan en las dos cooperativas.

Por otro lado, desde el punto de vista de actividades de vinculación por parte de las Instituciones de Educación Superior (IES) en el caso del Ecuador es una de las funciones sustantivas que en conjunto con la instrucción pedagógica y la pesquisa, tiende a solucionar los inconvenientes de la comunidad, por medio de proyectos, prácticas preprofesionales, capacitación y otros servicios profesionales en salud, que posibilitan la comunicación y la interacción de manera efectiva de la universidad con la sociedad, del mismo modo como la relación de la teoría con la práctica del quehacer de los universitarios en función del desarrollo social (11).

De hecho la Universidad Católica de Santiago de Guayaquil, viene ejecutando 19 proyectos de desarrollo comunitario que abordan temáticas vinculadas al campo profesional, entre las temáticas se encuentra el Proyecto "Familias y Comunidades Saludables" realizado por la Facultad de Ciencias Médicas, desarrollado en la Cooperativa 25 de Julio y Virgen del Cisne, ubicadas al noroeste de la ciudad de Guayaquil, en y a las faldas del cerro San Eduardo, con la finalidad de contribuir al mejoramiento de las capacidades necesarias de la familia para mejorar la cohesión como célula social básica, la salud, vivienda y entorno, donde el sujeto de atención se enfoca en la familia (12).

Por consiguiente la investigación planteada conlleva a la necesidad de identificar los factores de riesgos de la población de estudio, por lo que se planteó el objetivo de determinar la prevalencia de enfermedades neuro-músculo esqueléticas, en la Comunidad de San Eduardo de la Ciudad de Guayaquil, por medio del análisis de la base de datos del Proyecto Familias y Comunidades Saludables.

\section{MATERIALES Y MÉTODOS}

$\mathrm{S}$ e realizó un estudio retrospectivo que se llevó a cabo en el 2018, para el efecto se identificó a la familia, por datos de ubicación; rol que desempeñó el participante; tipo y composición familiar; riesgos individuales; antecedentes de patologías de carácter familiar e institucional y el perfil de los miembros de la Comunidad de San Eduardo, según factores de riesgo (13). 
El presente estudio tiene un alcance descriptivo y de corte transversal (14), porque el objetivo fue determinar la prevalencia de enfermedades neuromúsculo esqueléticas, en la Comunidad de San Eduardo-Guayaquil.

El proceso investigativo fue de carácter deductivo, porque permitió extraer conclusiones a partir de los resultados obtenidos de la presente investigación (15).

La recolección de los datos se ejecutó por medio de acciones y actividades de vinculación del proyecto "Familias y Comunidades Saludables", con intervención de las cinco carreras de la Facultad de Ciencias Médicas, Universidad Católica de Santiago de Guayaquil, conformada por Fisioterapia, Medicina, Nutrición, Enfermería y Odontología, En el año 2018, los estudiantes de diferentes ciclos y docentes de apoyo, que participaron en el estudio, se los capacitó en cada uno de los procedimientos. Se coordinó con líderes comunitarios, quienes afianzaron la comunicación del sector de población seleccionada.

La observación fue una de primeras técnicas a emplearse (16), utilizándose un mapa sectorizado de la zona, para dirigirse casa a casa, de los miembros que componen las familias de 729 hogares de Comunidad San Eduardo, para identificar posibles potenciales de riesgos relacionados con determinantes de la salud, así como, el conocimiento de formas de articulación social de actores presentes en la localidad, comportamientos, valores e intereses relativos al bienestar integral, formulándose a los participantes, preguntas claves, bajo el instrumento de riesgo familiar (RFT 5-33), avalado por vicerrectorado de vinculación.

Entre las variables que figuraron fueron: edad (años) según el sexo; frecuencia de enfermedades neuromúsculo esqueléticas; deporte, actividad física; dolor de espalda y/o articular, mediante parámetro Sí o No.

Los criterios de inclusión que se consideraron fueron: Personas de ambos sexos, con enfermedades neuromusculoesqueléticas, que habitaran en la Comunidad de San Eduardo.

\section{Análisis estadístico}

El análisis de la información se realizó utilizando una estadística descriptiva, específicamente la distribución porcentual, mediante el parámetro Si o No, para identificar principales características, presentándose por medio de gráficos $\mathrm{y}$ figuras.

\section{RESULTADO}

$\mathrm{S}$ e interpreta el rango de edad según el sexo, que predominó en los habitantes de la comunidad de San Eduardo, de 2.759 personas, correspondió un $75 \%(n=2069)$ a los mayores de 42 años y en un $25 \%(n=690)$ a los menores de 13 años; la edad promedio de la población es de 28,6 años; una mediana de 25 años y la máxima de 99 años. De los cuales $1.388(50,3 \%)$ fueron hombres $y$ $1.371(49,7 \%)$ mujeres que no representa diferenciación significativa con respecto al sexo de la población. (Gráfico 1). 


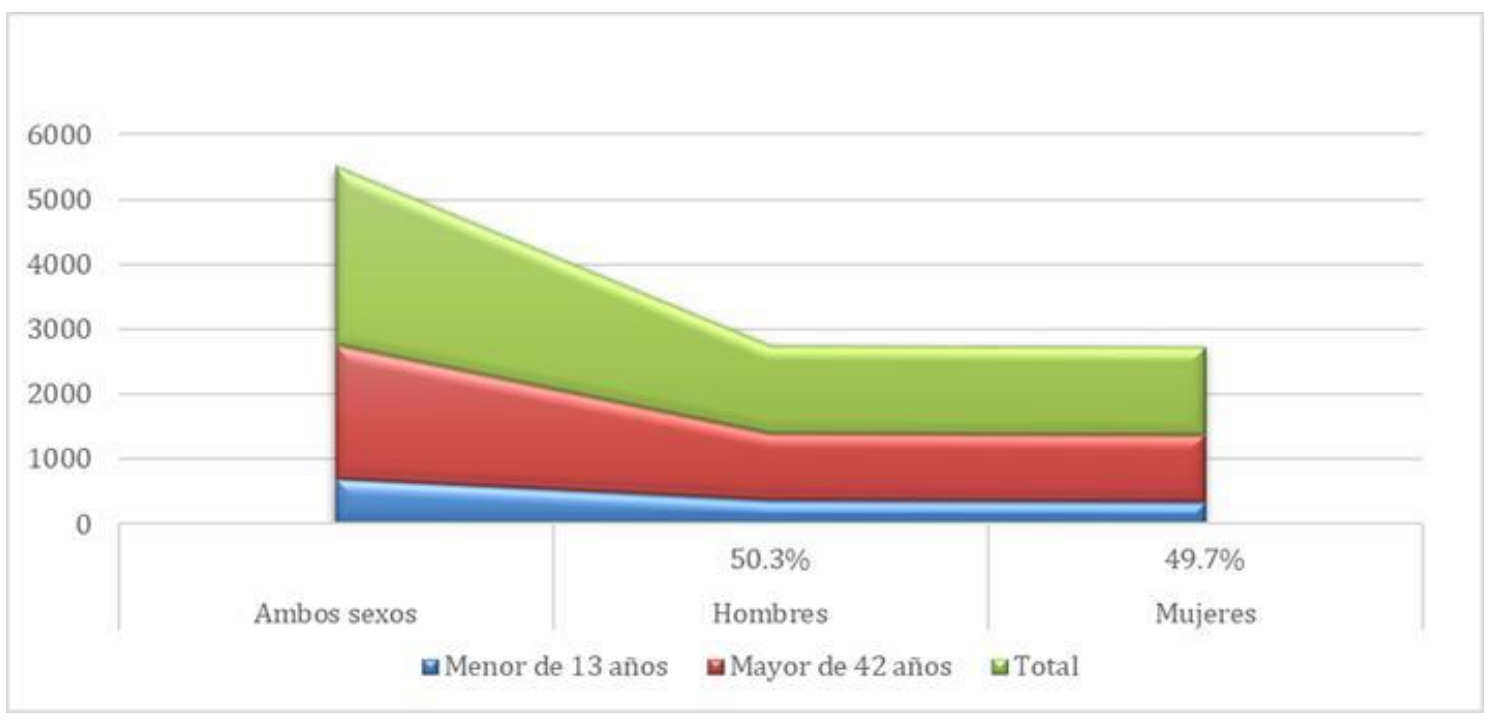

Gráfico 1. Distribución porcentual de sexo y edad.

Según las alteraciones neuro-músculo esqueléticas más frecuentes relacionadas con el estudio, se encontraron de un total de 729 participantes que respondieron SI, 194 personas con lumbalgia el $26 \%$; 117 con artritis con un $16 \%$; luego 103 con osteoporosis, el 14,1\% y por último 99 con artrosis que correspondieron en un 13,6\%.
Por otro punto, no prevalecieron casos a nivel del sistema nervioso periférico, pero si, trastornos neurológicos del sistema nervioso central, de los cuales 26 contestaron que Sí tenían enfermedad mental, seguido de epilepsia con 25 de un total de 51 personas. (Figura 1).

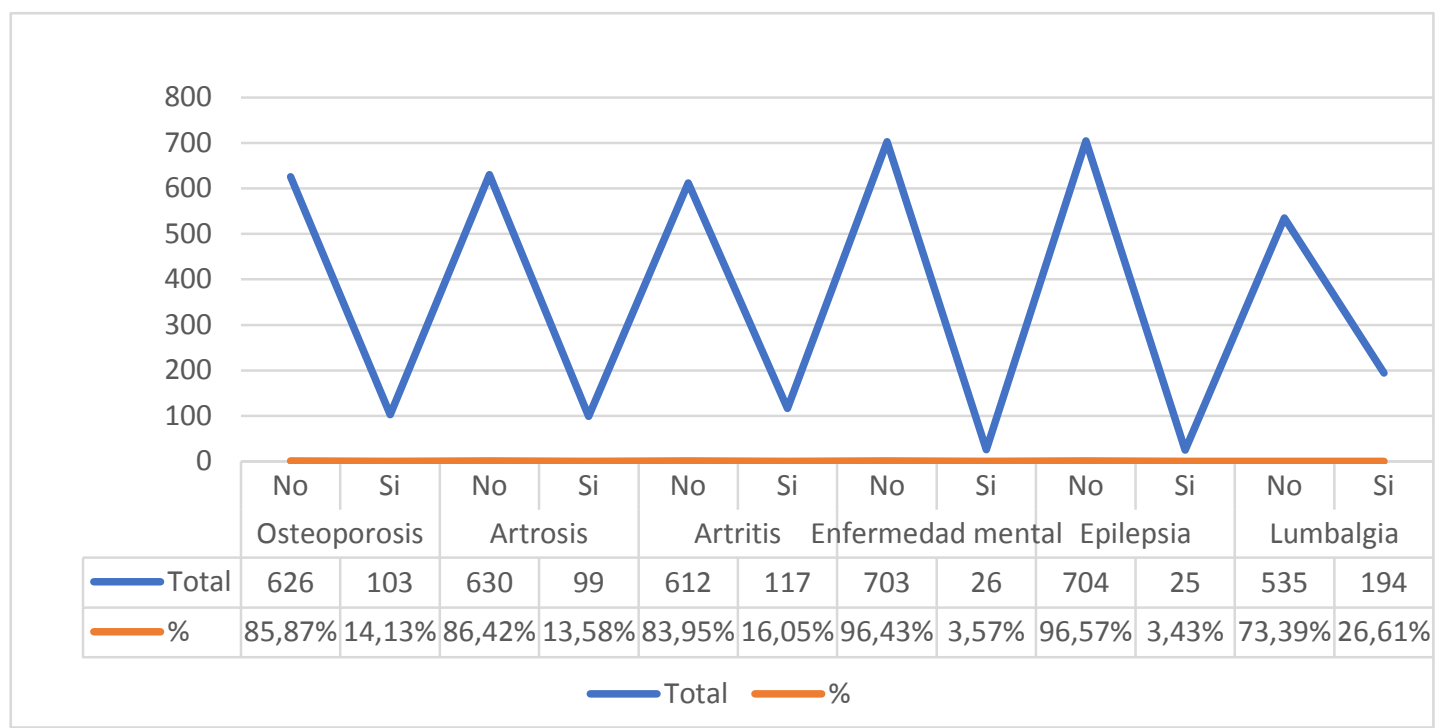

Figura 1. Enfermedades neuro-músculo esqueléticas, mediante parámetro Sí y No. 
De acuerdo a los resultados obtenidos el 33,4 \% de los participantes refirió dolor de espalda y/o articular en el último mes, considerándose un problema de salud, a causa de posturas laborales, cargas pesadas y movimientos repetitivos, predominantes en el desarrollo de enfermedades del sistema músculo esquelético. (Figura 2).

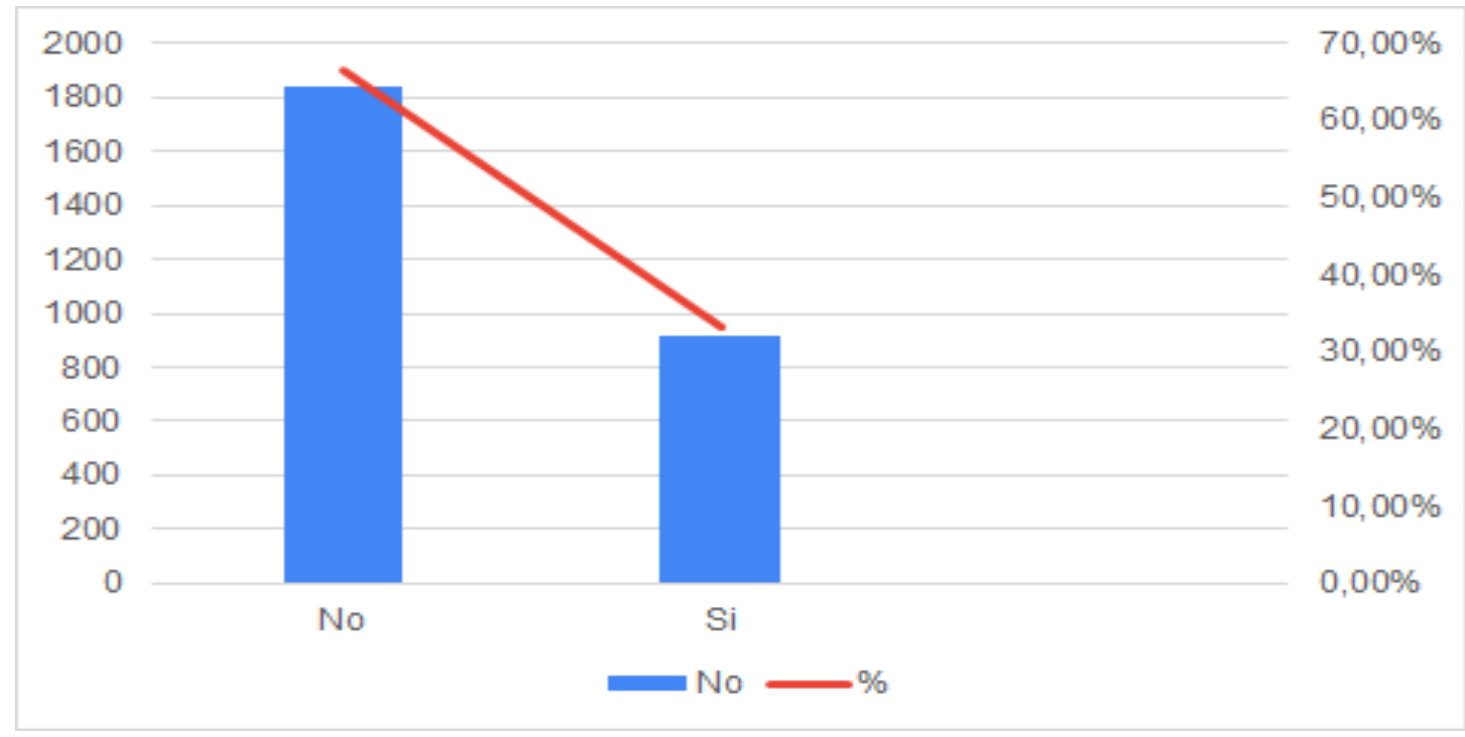

Figura 2. Prevalencia de dolor de espalda y/o articular, mediante parámetro Sí y NO.

De acuerdo con el estudio 1.891 participantes contestaron NO realizar actividad física o algún tipo de deporte. Siendo este detalle proporcional a los dolores de espalda que se refirió anteriormente. Se observa que 868 personas contestaron que Sí realizan algún tipo de ejercicio físico, siendo este grupo el adecuado, para estándares de vida saludable. (Figura 3).

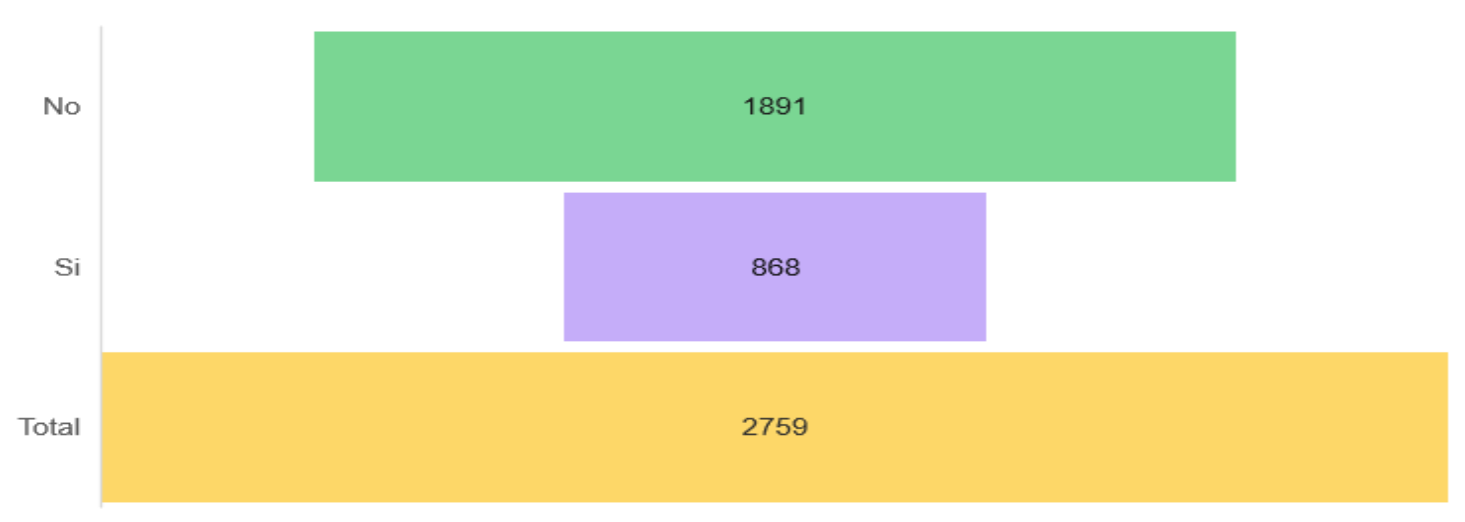

Figura 3. Escala de valor de la actividad física y deporte, mediante parámetro Sí y NO. 


\section{DISCUSIÓN}

De acuerdo a un estudio sobre la "Prevalencia de enfermedades neurológicas en un centro de atención primaria en el Cantón Suscal, Ecuador", en el año 2016, por los investigadores Moreno-Zambrano, Peñaherrera-Oviedo y Santibáñez-Vásquez, quienes analizaron que no habían datos disponibles que describieran la epidemiología de las enfermedades neurológicas, por la escasez de datos en el Ecuador (17). Cuyos resultados que se obtuvieron de dicha investigación se relacionaron con la que se realizó en la Comunidad de San Eduardo, al observarse una similitud en la baja prevalencia de las enfermedades.

Es la primera vez, que se realizó un estudio científico, en la Parroquia Tarqui, del Cantón Guayaquil, Provincia del Guayas, sobre la prevalencia de enfermedades neuro-músculo esqueléticas, en base a la información obtenida de cada uno de los miembros que componen las familias de 729 hogares, de la Comunidad de San Eduardo, Cooperativas "25 de Julio y Virgen del Cisne".

Se contribuyó al conocimiento de la prevalencia de enfermedades neuromúsculo esqueléticas, de los resultados obtenidos de la base de información del Proyecto Familias y Comunidades Saludables.

De acuerdo con los datos de la investigación, los trastornos neurológicos a nivel del sistema nervioso central, que prevalecieron fueron la enfermedad mental y epilepsia. Se analiza que las lesiones del sistema nervioso periférico no predominaron, porque en el instrumento RFT 5-33, de recopilación de la información, no se las consideró como antecedentes de carácter patológico personal y familiar. Además, se puede analizar según un estudio sobre enfermedades neuromusculares, realizado por Castiglion, Bevilacqua $\mathrm{y}$ Hervias (2015), que indicaron que es necesario mantener un alto nivel de sospecha clínica porque los signos y síntomas pueden ser sutiles, de lenta evolución y no ser referidos directamente por el paciente afectado (18).

Con relación a los síntomas en un 33,4 $\%$ de los participantes del estudio, refirieron dolor de espalda y/o articular en el último mes, analizándose que el factor de carga física y movimientos repetitivos, tienen una relevancia en la explicación de riesgo para presentar cambios en diferentes áreas corporales, un estudio que se relaciona con el trabajo de investigación realizado en Barraquilla-Colombia a trabajadores en empresas operativas, indicaron que la mayoría de los encuestados en cambio refirieron altas tasas de prevalencia para espalda baja en un 50,6\%; seguida por cuello el 13,2\%, siendo una similitud de ambos estudios por el origen de los síntomas osteomusculares relacionados a posturas biomecánicas inadecuadas y por el número de repeticiones (19).

Dentro de la población de estudio, la lumbalgia es un problema sanitario multifactorial, indicativo como principal síntoma de enfermedades neuro-musculo esqueléticas, al relacionarse con el dolor.

Se fomentó una cultura de promoción de la salud y de estrategias de prevención, por medio del Proyecto Familias y Comunidades Saludables, para la Comunidad de San Eduardo, que presentaron enfermedades neuro-músculo esqueléticas, para beneficio de la condición física/funcional, y disminución de factores de riesgos discapacitantes. 


\section{CONCLUSIÓN}

$\mathrm{S}$ e delimitó el grupo de población de estudio, de la prevalencia de enfermedades neuro-músculo esqueléticas, de cada uno de los miembros, que componen las familias de 729 hogares, de la Comunidad de San Eduardo, Cooperativas "25 de Julio y Virgen del Cisne", Parroquia Tarqui, Cantón Guayaquil, Provincia del Guayas.

Se pudo conocer que prevalecieron las enfermedades músculo esqueléticas de acuerdo al porcentaje de los participantes del estudio de investigación que contestaron $\mathrm{Si}$, predominando la lumbalgia, artritis, osteoporosis, artrosis y en menor proporción los trastornos neurológicos centrales relacionadas a la enfermedad mental y epilepsia.

De acuerdo con los datos obtenidos del trabajo de investigación, 921 participantes, refirieron dolor de espalda y/o articular en el último mes, coincidiendo al alto índice de la lumbalgia como signo de alarma que precede a diagnósticos de otras patologías neuro-músculo esqueléticas, ocasionadas por posturas mantenidas de manera estática, movimientos repetitivos, cargas pesadas; llegando este problema a desencadenar grandes complicaciones en el ámbito de la salud.

\section{REFERENCIAS BIBLIOGRÁFICAS}

1. Dzul-Gala F, Tun-Colonia J, ArankowskySandoval G, Pineda-Cortes J, SalgadoBurgos H, Pérez-Padilla E. Relación entre la sobrecarga y el índice depresivo de cuidadores primarios de pacientes con enfermedades neuromusculoesqueléticas [Internet]. [citado 3 de abril de 2021]. Disponible en:
bin/new/resumen.cgi?IDARTICULO=8227 7

2. Organización Mundial de la Salud. Trastornos musculoesqueléticos [Internet]. [citado 3 de abril de 2021]. Disponible en: https://www.who.int/es/news-room/factsheets/detail/musculoskeletal-conditions

3. Organización Mundial de la Salud. OMS ¿Qué son los trastornos neurológicos? [Internet]. WHO. [citado 3 de abril de 2021]. Disponible en: http://www.who.int/features/qa/55/es/

4. Medina AFS. Prevalencia de desórdenes músculo esqueléticos en trabajadores de una empresa de comercio de productos farmacéuticos. Rev Cienc salud. 24 de mayo de 2018; 16(2):203-18.

5. Vega-Hinojosa $\mathrm{O}$, Cardiel MH, OchoaMiranda P. Prevalencia de manifestaciones musculoesqueléticas y discapacidad asociada en una población peruana urbana habitante a gran altura. Estudio COPCORD. Estadio I. Reumatol Clin. 1 de septiembre de 2018; 14(5):278-84.

6. Alva-Díaz C, Huerta-Rosario A, PachecoBarrios K, Molina RA, Navarro-Flores A, Aguirre-Quispe $\mathrm{W}$, et al. Neurological diseases in Peru: a systematic analysis of the global burden disease study. Arquivos de Neuro-Psiquiatria. Mayo de 2020; 78(5):282-9.

7. Carvajal J, Galeano LM, Olarte D, Arboleda A, Restrepo A, Bareño J. Prevalencia de síndromes neuropsicológicos del adulto en una unidad de neuropsicología en Medellín, Colombia. Acta Neurol Colomb. 31 de marzo de 2015; 31(1):20-6.

8. España. Secretaria de Salud Laboral y Medio Ambiente. Trastornos músculoesqueléticos relacionados con el trabajo. Ministerio de Trabajo Migraciones y Seguridad Social; 2019.

9. Camacho, Esteban, J, Paradas C. Informe de la Fundación Del Cerebro sobre el impacto social de la esclerosis lateral 
amiotrófica y las enfermedades neuromusculares | Neurología [Internet]. [citado 3 de abril de 2021]. Disponible en: https://www.elsevier.es/es-revistaneurologia-295-avance-resumen-informefundacion-del-cerebro-sobreS0213485315000341

10. Agila-Palacios E, Colunga-Rodríguez $C$, González-Muñoz E, Delgado-García D. Síntomas Músculo-Esqueléticos en Trabajadores Operativos del Área de Mantenimiento de una Empresa Petrolera Ecuatoriana. Ciencia \&amp; trabajo. Diciembre de 2014; 16(51):198-205.

11. Polaino CJ, Romillo A de J. Vinculación con la Sociedad en la Universidad de Otavalo, Ecuador. Formación universitaria. 2017; 10(3):21-30.

12. Programas y proyectos de vinculación - UCSG [Internet]. [citado 3 de abril de 2021]. Disponible en: https://www.ucsg.edu.ec/vinculacion/pro gramas-y-proyectos-de-vinculacion/

13. Jaramillo L. Análisis Estadístico del Proyecto: «Familias y Comunidades Saludables». Guayaquil: Universidad Católica de Santiago de Guayaquil; 2018 p. 4-30.

14. Hernández Sampieri R, Fernández Collado C, Baptista Lucio P. Metodología de la investigación. México, D.F.: McGraw-Hill Education; 2014.

15. Arias F. G. El Proyecto de Investigación 6a Edición [Internet]. [citado 3 de abril de 2021]. Disponible en: https://www.researchgate.net/publicatio n/301894369

16. Baena Paz G. Metodología de la investigación (3a. ed.) [Internet]. $3 \circ$. Ciudad de México: Grupo Editorial Patria; 2017. 157 p. Disponible en:
http://www.biblioteca.cij.gob.mx/Archivo s/Materiales_de_consulta/Drogas_de_Abus o/Articulos/metodologia\%20de $\% 20 \mathrm{la} \% 2$ 0investigacion.pdf

17. Moreno-Zambrano D, PeñaherreraOviedo C, Santibáñez-Vásquez R. Prevalencia de enfermedades neurológicas en un centro de atención primaria en el Cantón Suscal, Ecuador. Rev Mex Neuroci. 15 de junio de 2016; 17(1):44-9.

18. Castiglioni $C$, Bevilacqua JA, Hervias KLGAC. Enfermedades neuromusculares en el adolescente. Síntomas y signos clínicos orientadores al diagnóstico. Revista Médica Clínica Las Condes. Enero de 2015; 26(1):66-73.

19. Castro C-CG Ardila-Pereira LC, OrozcoMuñoz Y del S, Sepúlveda-Lázaro EE, Molina-Castro. Factores de riesgo asociados a desordenes músculo esqueléticos en una empresa de fabricación de refrigeradores | Revista de Salud Pública [Internet]. [citado 3 de abril de 2021]. Disponible en: https://revistas.unal.edu.co/index.php/re vsaludpublica/article/view/57015

\footnotetext{
Limitaciones y fortalezas. Como los resultados logrados fueron obtenidos de manera factible, se hace justificable realizar en el futuro otros estudios retrospectivo, prospectivo y comparativo, con una visión amplia en el campo de la investigación.

Conflicto de intereses. Los autores declaran no tener conflicto de intereses para la publicación del presente artículo científico.

Financiamiento. Ninguno

Agradecimiento. A la coordinadora del Proceso de la Unidad de Titulación Estudiantil, UTE y Vicerrectorado de Vinculación de la Universidad Católica de Santiago de Guayaquil, por facilitar la base de datos del Proyecto "Familias y Comunidades Saludables".
} 